\title{
Identification of Novel Inhibitors against Coactivator Associated Arginine Methyltransferase 1 Based on Virtual Screening and Biological Assays
}

\author{
Fei Ye, ${ }^{1,2}$ Weiyao Zhang, ${ }^{1}$ Wenchao Lu, ${ }^{3,4}$ Yiqian Xie, ${ }^{3}$ Hao Jiang, ${ }^{3,4}$ Jia Jin, ${ }^{1}$ and Cheng Luo ${ }^{3}$ \\ ${ }^{1}$ College of Life Sciences, Zhejiang Sci-Tech University, Hangzhou, China \\ ${ }^{2}$ Key Laboratory of Plant Secondary Metabolism and Regulation of Zhejiang Province, Hangzhou, China \\ ${ }^{3}$ Drug Discovery and Design Center, State Key Laboratory of Drug Research, Shanghai Institute of Materia Medica, \\ Chinese Academy of Sciences, Shanghai, China \\ ${ }^{4}$ University of Chinese Academy of Sciences, Beijing, China
}

Correspondence should be addressed to Fei Ye; yefei@zstu.edu.cn and Jia Jin; aukaukauk@163.com

Received 2 August 2016; Revised 19 September 2016; Accepted 3 October 2016

Academic Editor: You-Lin Tain

Copyright $\odot 2016$ Fei Ye et al. This is an open access article distributed under the Creative Commons Attribution License, which permits unrestricted use, distribution, and reproduction in any medium, provided the original work is properly cited.

Overexpression of coactivator associated arginine methyltransferase 1 (CARM1), a protein arginine N-methyltransferase (PRMT) family enzyme, is associated with various diseases including cancers. Consequently, the development of small-molecule inhibitors targeting PRMTs has significant value for both research and therapeutic purposes. In this study, together with structure-based virtual screening with biochemical assays, two compounds DC_C11 and DC_C66 were identified as novel inhibitors of CARM1. Cellular studies revealed that the two inhibitors are cell membrane permeable and effectively blocked proliferation of cancer cells including HELA, K562, and MCF7. We further predicted the binding mode of these inhibitors through molecular docking analysis, which indicated that the inhibitors competitively occupied the binding site of the substrate and destroyed the protein-protein interactions between CARM1 and its substrates. Overall, this study has shed light on the development of small-molecule CARM1 inhibitors with novel scaffolds.

\section{Introduction}

Arginine methylation is an important posttranslational modification catalyzed by protein arginine $\mathrm{N}$-methyltransferases (PRMTs) [1, 2]. During PRMT catalysis, the methyl group of $\mathrm{S}$-adenosyl-L-methionine (AdoMet, SAM) is transferred to the guanidino group of the target arginine, resulting in monoor dimethylated arginine residues along with S-adenosylL-homocysteine (AdoHcy, SAH) as a coproduct [3]. There are nine PRMTs identified so far, which can be classified into three categories: type I (PRMT1, 2, 3, 4, 6, and 8), type II (PRMT5 and 9) and type III (PRM7) [4]. Type I PRMTs catalyze mono- and asymmetric dimethylation of arginine residues, whereas type II PRMTs catalyze mono- and symmetric dimethylation of arginine residues [5]. PRMT7 is the only known type III PRMT, which catalyzes monomethylation of arginine [6].
PRMT4, also known as CARM1 (coactivator associated arginine methyltransferase 1) methylates a wide variety of histone and nonhistone substrates including H3R17, H3R26 [7], SRC-3 [8], CBP/p300 [9], NCOA2 [10], PABP1 [11], and $\mathrm{SmB}$ [12]. Consequently, CARM1 participates in many cellular processes by impacting chromatin architecture and transcriptional initiation $[9,13]$, RNA processing and stability [14], and RNA splicing [12]. Overexpression of CARM1 has been observed in multiple cancer types including myelocytic leukemia [15] and breast [10], prostate [16], lung [17], and colorectal carcinomas [18], making it a potential target for anticancer therapy.

Due to essential roles of CARM1 in the regulation of cellular functions as well as tumorigenesis, discovery of CARM1 inhibitors has recently attracted much attention. To date, a number of CARM1 inhibitors have been reported [19-27] 
(see Figure S1 in Supplementary Material available online at http://dx.doi.org/10.1155/2016/7086390). According to the chemical structures, these inhibitors can be divided into several categories: (i) 3,5-bis(bromohydroxybenzylidene) piperidin-4-one inhibitors (compounds 1-2 in Figure S1), (ii) pyrazole inhibitors (compounds 3-10 in Figure S1), (iii) benzo $[d]$ imidazole inhibitors (compounds $11-13$ in Figure S1), and (iv) other inhibitors (compounds 14-15 in Figure S1) [28]. However, the majority of these inhibitors are lacking selectivity and drug-likeness; thus turning these inhibitors into therapeutically useful compounds is challenging. Therefore, it is still of significant interest to discover selective inhibitors targeting CARM1 with good pharmacological properties.

Virtual screening is an important approach for leadcompound discovery and has been successfully used in multiple projects $[29,30]$. Recently, several crystal structures of CARM1 were determined, providing a prerequisite for structure-based virtual screening [26, 31-33]. Herein, due to the convenience and low cost of this approach, docking-based virtual screening was utilized to identify novel inhibitors of CARM1 from the Specs database (http://www.specs.net/). The candidates selected by virtual screening were then tested by biochemical experiments and eventually two novel inhibitors of CARM1 were identified. Among them, the more potent inhibitor DC_C66 displayed selectivity against PRMT1, PRMT6, and PRMT5. Molecular docking was conducted to investigate the binding modes of these inhibitors and molecular basis of selectivity for CARM1. Furthermore, cellular studies revealed that both inhibitors exhibited antiproliferation activity in several CARM1-associated cancer cell lines. Overall, this study has provided chemical probes in exploring biological functions of CARM1 and information for further optimization of potent inhibitors.

\section{Materials and Methods}

\subsection{Virtual Screening Protocol}

2.1.1. Protein Preparation. The crystal structure of CARM1 in complex with indole inhibitor (PDB code 2Y1W) was used as a target for subsequent virtual screening [26]. The water molecules and ions were initially removed. The protein status was optimized through the Protein Preparation Wizard Workflow provided in the Maestro [34], with a $\mathrm{pH}$ value of $7.0 \pm 2.0$. Other parameters were set as the default. Residues within a distance of $6 \AA$ around indole inhibitor were defined as binding pocket.

2.1.2. Ligand Database Preparation. The Specs database (http://www.specs.net/), containing 287,000 compounds, was utilized for the virtual screening. To refine the database, we filtered it by Lipinski's rule of five [35] and removed pan-assay interference compounds (PAINS) [36-38] with Pipeline Pilot, version 7.5 (Accelrys Inc., San Diego, CA, USA) [39], yielding a database of around 180,000 smallmolecule compounds. The remaining molecules were treated by LigPrep [40] to generate all stereo isomers and different protonation states with Epik.

2.1.3. Virtual Screening Protocol. The virtual screening protocol is shown in Figure 1. Firstly, the energy scoring function of DOCK4.0 was used to dock the compound library into the defined binding site. The top-ranked 10500 candidates selected by DOCK4.0 were further evaluated and ranked by the AutoDock4.0 program, leading to a list of 1500 compounds. The program Glide 5.5 [41] in XP mode [42] was run to calculate the free binding energy between these 1500 compounds and CARM1 protein. In order to ensure diversity in the candidates, the top 300 compounds from Glide 5.5 were classified to 30 groups by SciTegic functional class fingerprints (FCFP_4) in Pipeline Pilot, version 7.5 (Accelrys Inc., San Diego, CA, USA) [39], and 1-3 compounds were picked from each group. Finally, 57 compounds were selected and purchased for biological evaluation.

2.2. Similarity-Based Analog Searching. According to the results of the biological tests, we used the compound DC_C11 to run a two-dimensional similarity search through the prepared Specs database using Similarity Filter from File in Pipeline Pilot, version 7.5 (Accelrys Inc., San Diego, CA, USA). We purchased 10 compounds and tested their biological activity towards CARM1.

2.3. In Vitro CARM1 Enzyme Inhibition and Selectivity Assay. The enzymatic inhibitory activities of compounds were measured by the AlphaLISA assay provided by Shanghai ChemPartner Co., Ltd. The compounds selected from virtual screening were transferred to the assay plate (white opaque OptiPlate-384, PerkinElmer). $5 \mu \mathrm{L}$ of enzyme solution (final concentration was $0.1 \mathrm{nM}$ ) or $\mathrm{pH} 8.0$ tris-based assay buffer (for Min well) was added to the assay plate and then centrifuged at $1000 \mathrm{rpm}$ for $1 \mathrm{~min}$. Afterwards, the assay plate was incubated for $15 \mathrm{~min}$ at room temperature (RT). Then $5 \mu \mathrm{L}$ of biotinylated $\mathrm{H} 3$ peptide/SAM mix (final concentrations were $50 \mathrm{nM}$ and $300 \mathrm{nM}$, resp.) was added to the assay plate, which was covered with TopSeal-Afilm and incubated for $1 \mathrm{~h}$ at RT after centrifuging at $1000 \mathrm{rpm}$ for $1 \mathrm{~min}$ (DMSO final concentration 1\%). Next, $5 \mu \mathrm{L}$ of acceptor beads (final concentration was $10 \mu \mathrm{g} / \mathrm{mL}$ ) was added to stop the enzymatic reaction. After incubating at room temperature for $60 \mathrm{~min}$, $10 \mu \mathrm{L}$ of donor beads was added (final concentration was $10 \mu \mathrm{g} / \mathrm{mL}$ ) in subdued light and then centrifuged at $1000 \mathrm{rpm}$ for $1 \mathrm{~min}$. Finally, the mixtures were incubated for $30 \mathrm{~min}$ at RT, and the signal was read in alpha mode using EnVision readers. The $\mathrm{IC}_{50}$ values were calculated by fit inhibition rates under different concentrations into GraphPad Prism 5.0 software.

2.4. Cell Viability Assay. The three cell lines, HELA, K562, and MCF7, were purchased from the American Type Culture Collection (ATCC). HELA, K562, and MCF7 were cultured in DMEM (Life Technologies) supplemented with 10\% FBS. All of the cell lines were seeded into 96-well plates at an appropriate density and then treated with compounds 


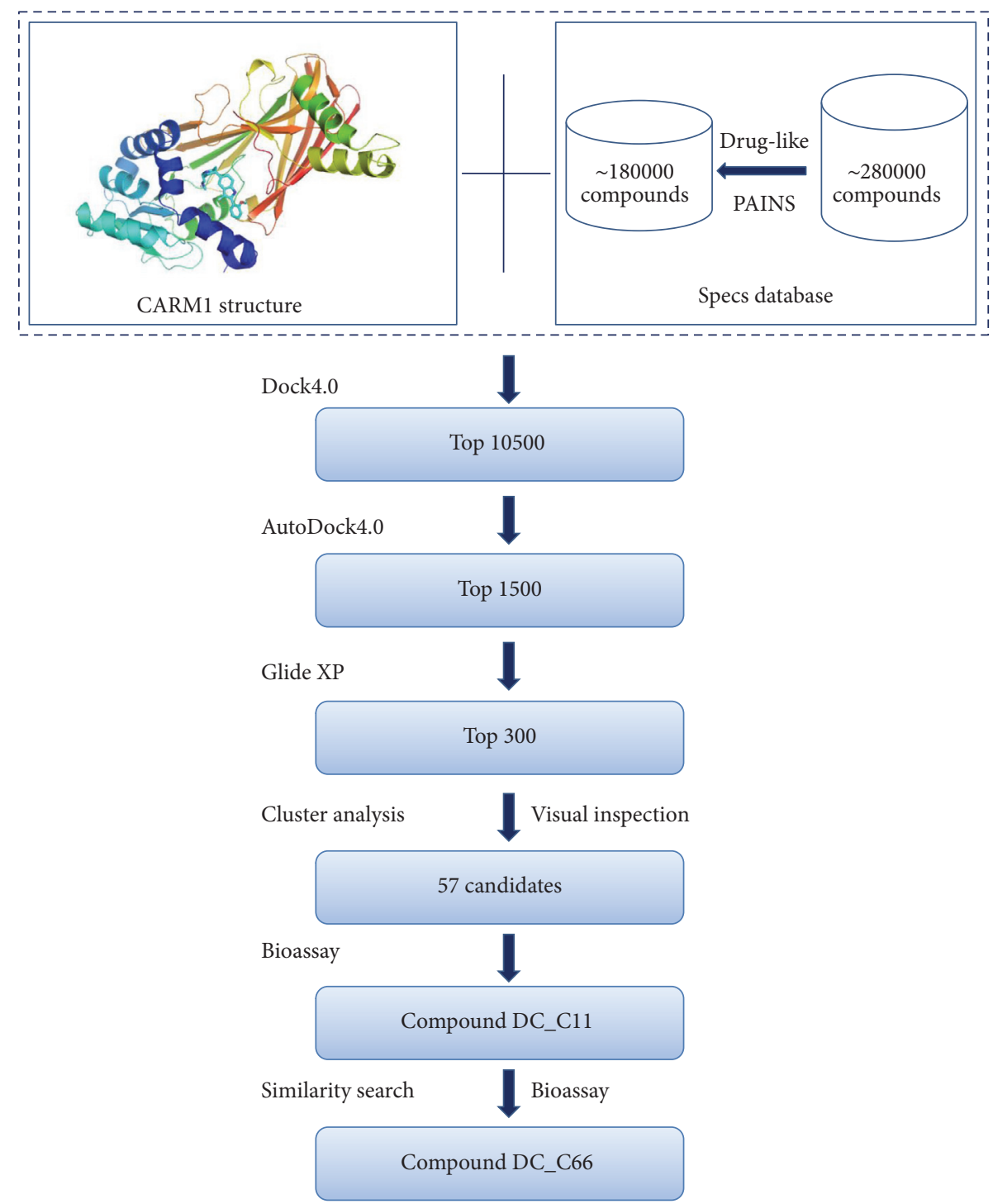

FIGURE 1: Flowchart of virtual screening procedures for CARM1 inhibitors.

of different concentrations or DMSO control. After $24 \mathrm{hrs,}$ $48 \mathrm{hrs}$, and $72 \mathrm{hrs}$, cell viabilities were measured by the MTT assay.

2.5. Binding Energy Calculations. In order to investigate the binding mode of DC_C11 and DC_C66, molecular docking was performed using Glide 5.5 in XP mode. The generated conformations were then used for binding energy calculations by Prime MM-GBSA (Molecular Mechanics/Generalized Born Surface Area method) [43]. The binding energy was calculated as follows:

$$
\begin{aligned}
\Delta G= & \left.E \_ \text {complex (minimized }\right) \\
& \left.-(E \text { ligand (minimized })+E \_ \text {receptor }\right) .
\end{aligned}
$$

In the calculations, the protein flexibility was set to $12 \AA$.

\section{Results and Discussion}

3.1. Structure-Based Virtual Screening. In this study, dockingbased virtual screening was performed to identify CARM1 inhibitors with novel scaffolds, and the flowchart is shown in Figure 1. The crystal structure of CARM1 in complex with indole inhibitor (PDB code $2 \mathrm{Y} 1 \mathrm{~W}$ ) was used as a target for the following in silico screening [26]. Residues within a distance of $6 \AA$ around indole inhibitor were defined as binding pocket, which contains the binding site of AdoMet and the arginine substrate. The Specs database (http://www.specs.net/), containing 287,000 compounds, was utilized for the virtual screening. To refine the database, we filtered it by Lipinski's rule of five and removed panassay interference compounds (PAINS) [36-38] with Pipeline Pilot, version 7.5 (Accelrys Inc., San Diego, CA, USA) [39], yielding a database of around 180,000 small-molecule compounds, which were subsequently docked and ranked with 
TABLE 1: Chemical structures and inhibitory activity $\left({ }^{\mathrm{a}} \mathrm{IC}_{50}, \mu \mathrm{M}\right)$ of selected compounds based on virtual screening against CARM1 and several other PRMTs.

Compound ID Specs ID

${ }^{\mathrm{a}}$ All assays were conducted in duplicate.

different score functions. The top-ranked 10500 candidates selected using energy scoring function of DOCK4.0 [44] were subsequently evaluated and ranked by the AutoDock 4.0 program [45], yielding a list of 1500 compounds. Then, the program Glide 5.5 (XP mode) [42] was chosen to calculate the free energy of binding between these 1500 compounds and CARM1 protein. According to the docking scores, the top-ranked 300 were clustered using Pipeline Pilot to ensure the scaffold diversity in the primary hits. The clustered molecules were cherry-picked by visual inspection based on the following considerations. (1) At least one compound was selected in each clustered group. (2) The binding modes were reasonable and molecules not occupying the SAM or substrate binding pocket were not chosen. (3) Among a group of similar molecules, compounds with lower molecular weight were preferred. Finally, 57 compounds were purchased for further biochemical validation.

3.2. Enzyme Inhibition and Selectivity Assay. All of the selected 57 candidate molecules were tested for CARM1 inhibition to determine their biochemical activities. Here, AlphaLISA assay, which is a powerful and versatile platform, was performed to test the inhibitory activities of the compounds. The enzyme solution and compounds or assay buffer were transferred to assay plates, which was incubated at RT. Then $5 \mu \mathrm{L}$ of biotinylated $\mathrm{H} 3$ peptide/SAM mix was added and incubated for $1 \mathrm{~h}$ at RT. Afterwards, acceptor and donor beads were added sequentially. The end point was read in alpha mode using EnVision readers, and $\mathrm{IC}_{50}$ values were calculated in GraphPad Prism 5.0 software. Among these candidates, only one compound DC_C11 was found to be active for CARM1 inhibition, which showed an $\mathrm{IC}_{50}$ value of $15 \mu \mathrm{M}$ (Table 1 ). We used this core structure as a hit to perform a two-dimensional similarity search through the
Specs database by Pipeline Pilot, version 7.5 (Accelrys Inc., San Diego, CA, USA) [39], leading to a compound DC_C66 which displayed inhibitory better potency for CARM1 with $\mathrm{IC}_{50}$ values of $1.8 \mu \mathrm{M}$.

To investigate the selectivity of the compounds, we tested the inhibitory activities of compounds DC_C11 and DC_C66 against several selected members of type I PRMT family, including PRMT1 and PRMT6 (Table 1). It was seen that DC_C66 showed relatively weaker activity against PRMT1 and PRMT6. Moreover, DC_C66 also showed little inhibitory activity of PRMT5, a member of type II PRMT, by $<50 \%$ inhibition rate at a concentration of $50 \mu \mathrm{M}$. Taken together, these results indicated that DC_C66 has a good selectivity for CARM1 against other selected PRMTs.

3.3. Cell-Based Activity. It has been reported that CARM1 was a potential target in many cancers; thus it is well accepted that inhibiting CARM1 could affect cancer cell proliferation. In this study, three human tumor cell lines including HELA (cervical cancer), K562 (myeloid leukemia), and MCF7 (breast cancer) were chosen to evaluate the cellular activity of the two compounds DC_C11 and DC_C66 in vivo. Sinefungin, a pan-PRMTs inhibitor which has the same scaffold as the cofactor SAM does, was evaluated for control experiment [46]. As shown in Figure 2, both DC_C11 and DC_C66 could inhibit proliferation of cancer cells in a time-dependent and dose-dependent manner while Sinefungin presented weaker inhibitory activity in cellular level. In the three cell lines, DC_C66 presents better antiproliferative cellular activity, which is consistent with their inhibitory activity in vitro. Combined with the biological data in vitro, we confirmed that compounds DC_C11 and DC_C66 are cell membrane permeable, which presented promising activity both in vitro and in cellular environment. 


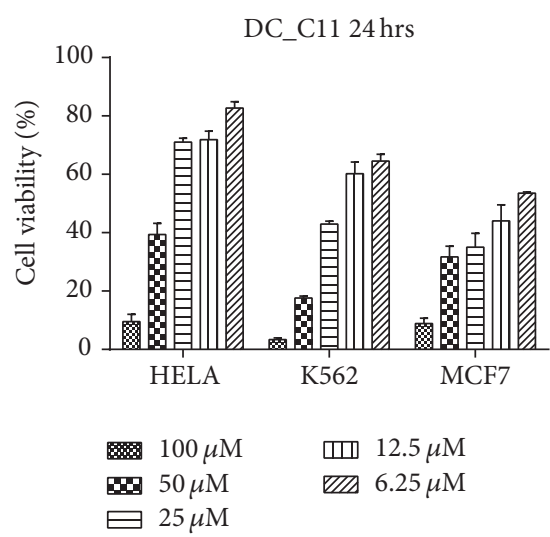

(a)

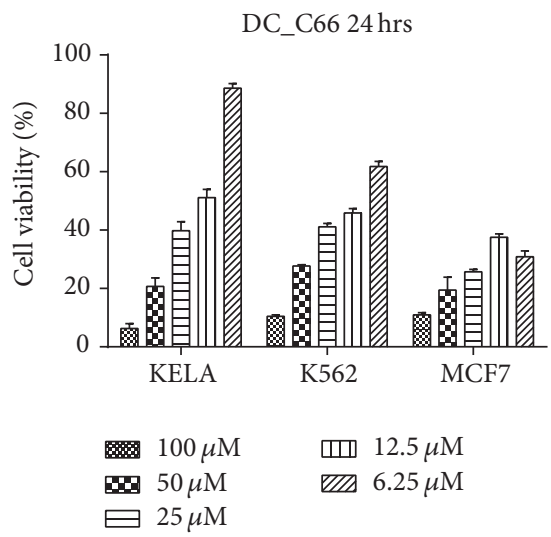

(d)

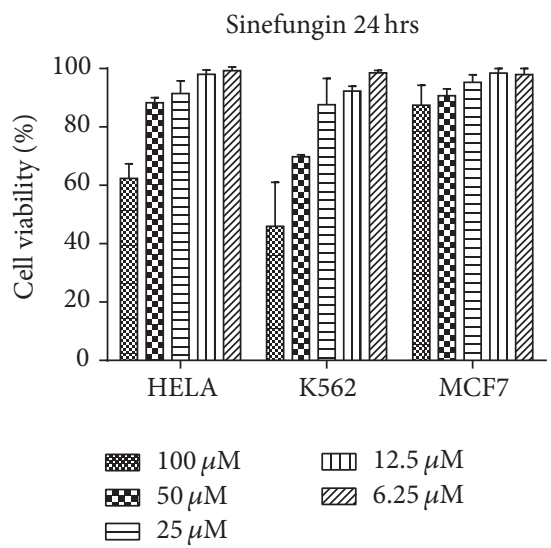

(g)

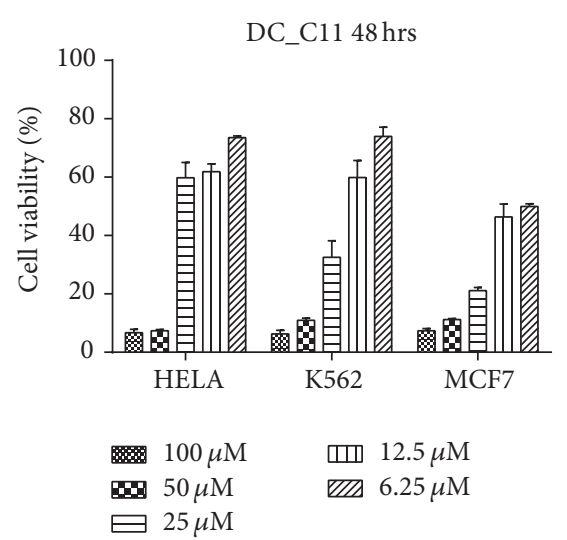

(b)

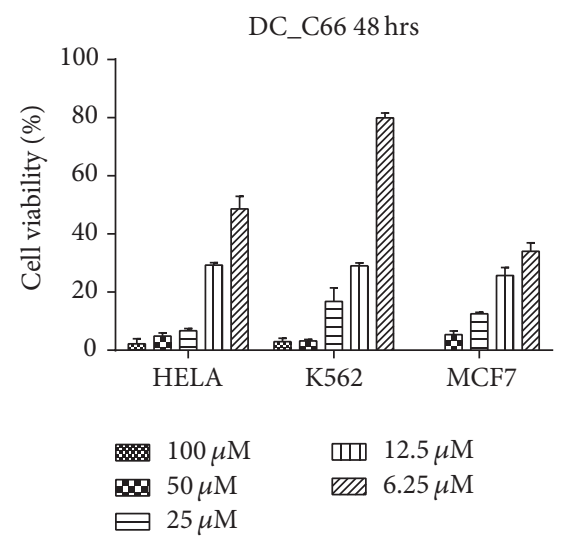

(e)

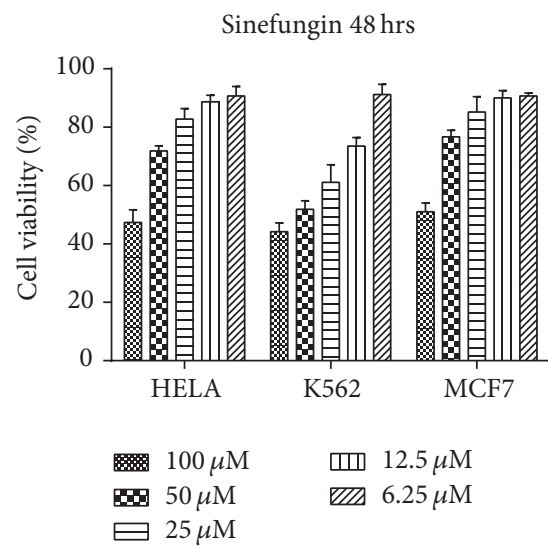

(h)

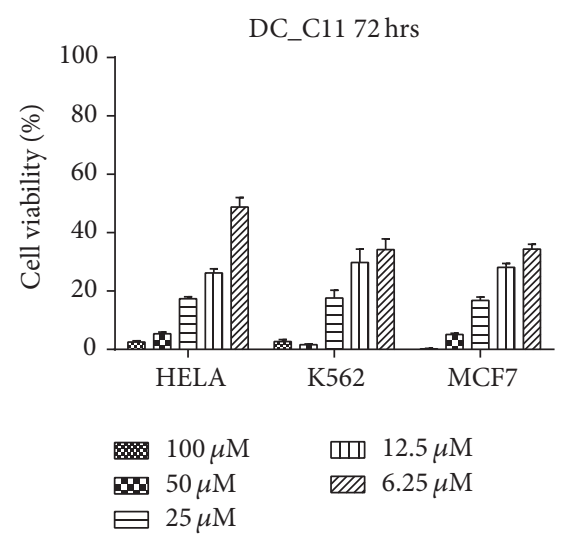

(c)

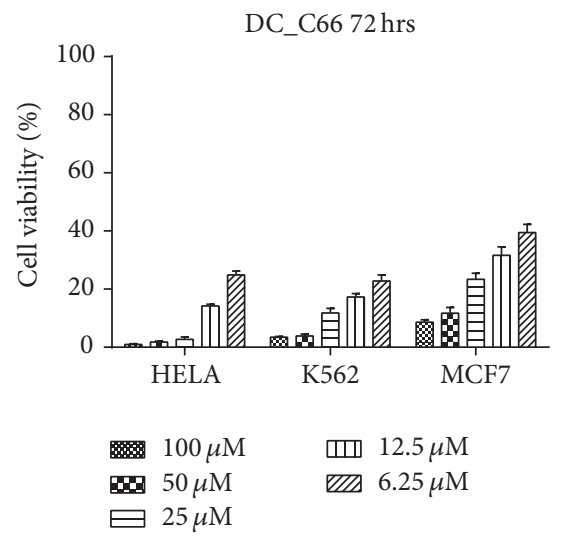

(f)

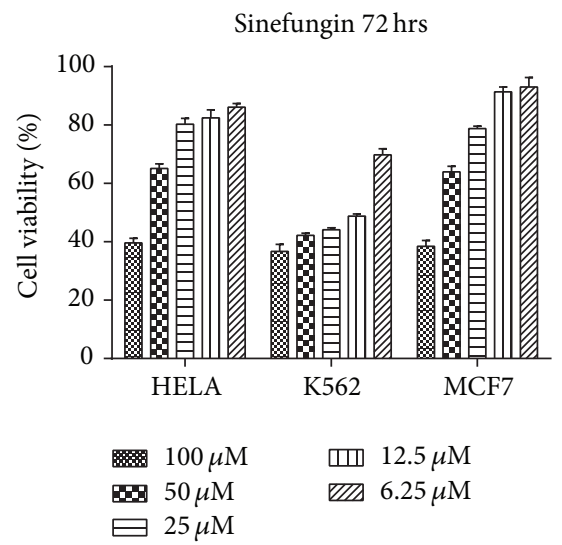

(i)

Figure 2: Antiproliferative effect of DC_C11 and DC_C66 on several cancer cell lines. (a-c) Time-dependent and dose-dependent inhibitory effect of DC_C11 on HELA, K562, and MCF7 within $24 \mathrm{hrs,} 48 \mathrm{hrs}$, and $72 \mathrm{hrs,} \mathrm{respectively.} \mathrm{(d-f)} \mathrm{Time-dependent} \mathrm{and} \mathrm{dose-dependent}$ inhibitory effect of DC_C66 on HELA, K562, and MCF7 within 24 hrs, 48 hrs, and 72 hrs, respectively. (g-i) Time-dependent and dosedependent inhibitory effect of Sinefungin on HELA, K562, and MCF7 within $24 \mathrm{hrs,} 48 \mathrm{hrs,} \mathrm{and} 72 \mathrm{hrs,} \mathrm{respectively.}$

3.4. Binding-Mode Analysis. To further understand the possible binding mode of DC_C11 and DC_C66 with CARM1, molecular docking study was performed with Glide in XP mode. As shown in Figure 3(a), both of DC_C11 and DC_C66 fit into the negative-charged binding pocket of substrate arginine in $\mathrm{H} 3$ peptide [33], implying that the compounds inhibit the activity of CARM1 by destroying the protein-protein interactions between CARM1 and substrate peptide. The phenyl ring bulks of DC_C11 and DC_C66 establish hydrophobic interactions with Y150, F153, Y154, N162, M163, and F475 in active site; the majority of these residues participate in interactions between CARM1 and its substrates (Figure 3) [33]. Besides, DC_C66 forms hydrogen bond with Y262 which probably accounts for its ability 


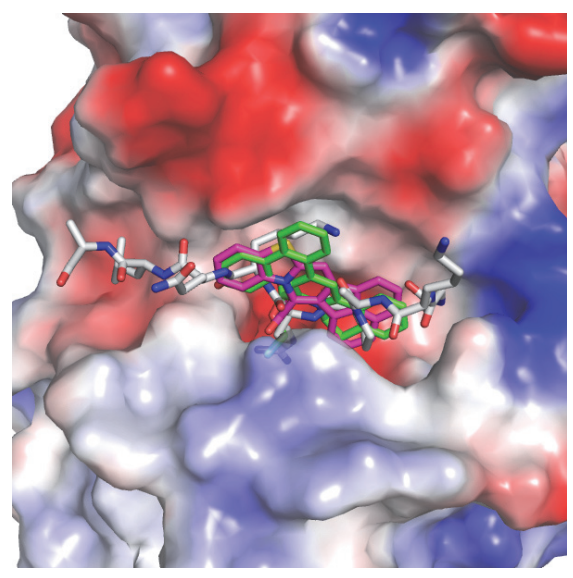

(a)

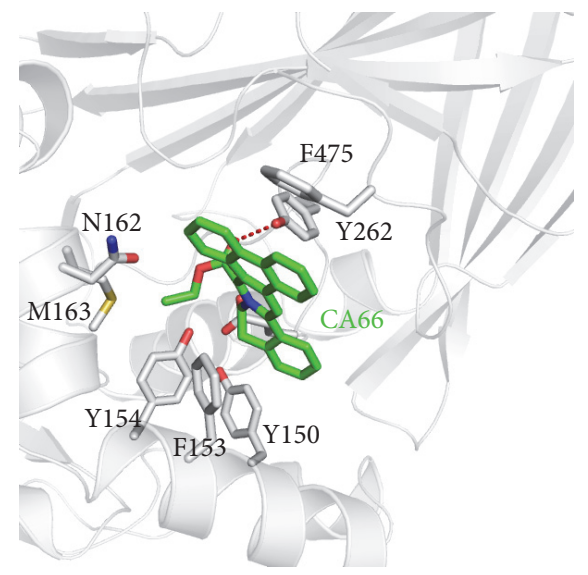

(b)

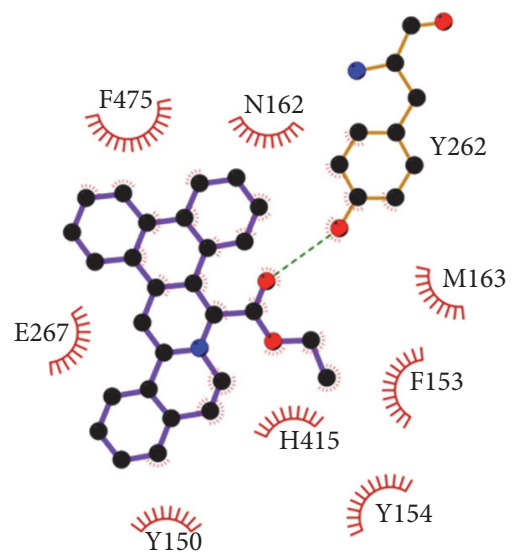

(c)

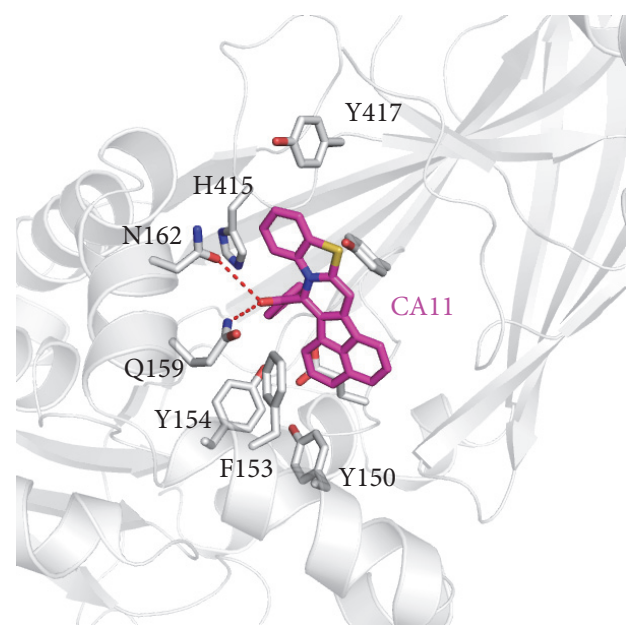

(d)

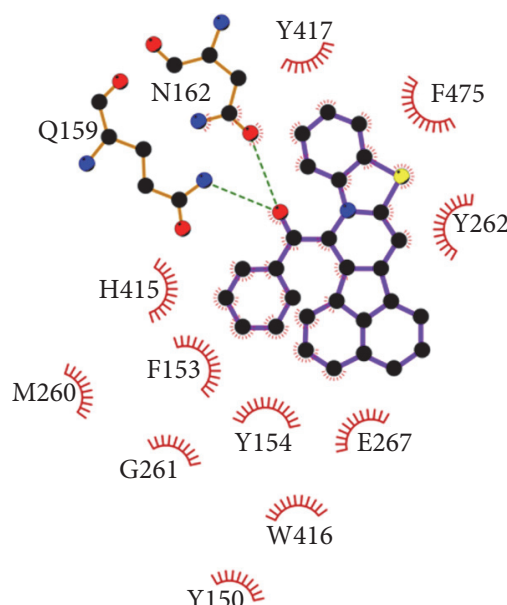

(e)

FIGURE 3: Predicted binding mode of DC_C11 and DC_C66 with CARM1 from docking analysis. (a) Superimposition of the binding modes of the two compounds and substrate H3 peptide (PDB ID: 5DX0). The structure of CARM1 is displayed in vacuum electrostatics. H3 peptide is shown as gray sticks, DC_C11 is shown as magenta sticks, and DC_C66 is displayed as green sticks. (b) A close view of the interactions between DC_C66 and CARM1 in the binding pocket; the key residues are shown as sticks. (c) Schematic diagram showing putative interactions between CARM1 and DC_C66. Residues involved in the hydrophobic interactions are shown as starbursts, and hydrogen-bonding interactions are denoted by dotted green lines. (d) A close view of the interactions between DC_C11 and CARM1 in the binding pocket; the key residues are shown as sticks. (e) Schematic diagram showing putative interactions between CARM1 and DC_C11.

TABLE 2: Binding energy for compounds DC_C66 and DC_C11.

\begin{tabular}{lcc}
\hline Compound ID & DC_C66 & DC_C11 \\
\hline Binding energy $(\mathrm{kcal} / \mathrm{mol})$ & -34.71 & -26.72 \\
\hline
\end{tabular}

to inhibit CARM1 activity (Figures 3(b) and 3(c)). Polar interactions between the oxygen in the carbonyl group of DC_C11 and side chain of Q159 as well as N162 also occur (Figures 3(d) and 3(e)). We further calculate binding energies of two compounds using Prime MM-GBSA [47] (Table 2). The results showed that $\mathrm{DC}_{-} \mathrm{C} 11$ binds to the substrate binding pocket with lower binding energy $(-26.72 \mathrm{kcal} / \mathrm{mol})$, followed by DC_C66 with a higher value $(-34.71 \mathrm{kcal} / \mathrm{mol})$. The calculated binding energies are in accordance with that of activity, rationalizing our experimental data of bioassays.
The sequence alignment and structural superposition of CARM1, PRMT1, and PRMT6 reveal several differences between these proteins (Figures S2 A-B), which may contribute to selectivity of the CARM1 inhibitors. In the $\mathrm{N}$ terminal helix, which is disordered in the crystal structure of rat PRMT1 and is essential for the enzymatic activity [48], the corresponding residues of F153 in CARM1 are S39 in PRMT1 and C50 in PRMT6 (Figures S2 A-B). Besides, F475 in C-terminal of CARM1 corresponds to R353 in PRMT1 and E374 in PRMT6. Since F153 and F475 are important components of the hydrophobic pocket that accommodates the phenyl ring bulk of DC_C66 (Figure 3), substitutions of the phenylalanine with hydrophilic amino acids may decrease the binding affinity of the CARM1 inhibitors (Figures S2 AB). These comparisons theoretically explain the selectivity of DC_C66 against CARM1 from the molecular basis. 


\section{Conclusion}

Posttranslational modifications of proteins have been increasingly recognized as essential modulators to their function in cells. In particular, arginine methylation, an important posttranslational modification, is catalyzed by PRMTs. CARM1, a member of PRMTs, has been implicated in a variety of cancers. Thus, the identification of selective inhibitors of CARM1 as probes to investigate CARM1 cellular function and its relevance in disease would be of significant interest in the field of epigenetics. Here in our study, by combining structure-based virtual screening and biochemical assays, we have identified DC_C11 and DC_C66 as novel inhibitors of CARM1, with $\mathrm{IC}_{50}$ values of 15 and $1.8 \mu \mathrm{M}$, respectively. Notably, DC_C66 displayed good selectivity against PRMT1, PRMT6, and PRMT5. The binding-mode prediction revealed that the two compounds can efficiently bind in the substrate binding site of CARM1 and thus inhibit the enzymatic activity by destruction of protein-protein interactions between CARM1 and its various substrates. Furthermore, the two compounds showed good cell permeability and blocked the proliferation of several cancer cells related to CARM1 overexpression. Overall, this study demonstrated an efficient docking-based virtual screening procedure that can be used to identify novel CARM1 inhibitors. These results paves the way for further development of inhibitors with novel scaffolds and functional probes to target CARM1 on the cellular level for both biological and therapeutic purposes.

\section{Competing Interests}

The authors declare no competing interests regarding the publication of this paper.

\section{Authors' Contributions}

Fei Ye and Weiyao Zhang contributed equally to this paper.

\section{Acknowledgments}

The authors gratefully acknowledge financial support from Zhejiang Province Natural Science Foundation (LQ14H 300003), The National Natural Science Foundation of China (81402849), Public Projects of Zhejiang Province (2015C33159 and 2016C31017), Zhejiang Provincial Top Key Discipline of Biology, Science Foundation of Zhejiang Sci-Tech University (ZSTU) under Grants no. 13042163-Y and no.13042159-Y, and the 521 Talent Cultivation Plan of Zhejiang Sci-Tech University.

\section{References}

[1] M. T. Bedford and S. G. Clarke, "Protein arginine methylation in mammals: who, what, and why," Molecular Cell, vol. 33, no. 1, pp. 1-13, 2009.

[2] M. T. Bedford and S. Richard, "Arginine methylation: an emerging regulator of protein function," Molecular Cell, vol. 18, no. 3, pp. 263-272, 2005.
[3] H. W. Lee, S. Kim, and W. K. Paik, "S-adenosylmethionine: protein-arginine methyltransferase. Purification and mechanism of the enzyme," Biochemistry, vol. 16, no. 1, pp. 78-85, 1977.

[4] M. T. Bedford, "Arginine methylation at a glance," Journal of Cell Science, vol. 120, no. 24, pp. 4243-4246, 2007.

[5] Y. Yang and M. T. Bedford, "Protein arginine methyltransferases and cancer," Nature Reviews Cancer, vol. 13, no. 1, pp. 37-50, 2013.

[6] Y. Feng, R. Maity, J. P. Whitelegge et al., "Mammalian protein arginine methyltransferase 7 (PRMT7) specifically targets RXR sites in lysine- and arginine-rich regions," Journal of Biological Chemistry, vol. 288, no. 52, pp. 37010-37025, 2013.

[7] B. T. Schurter, S. S. Koh, D. Chen et al., "Methylation of histone H3 by coactivator-associated arginine methyltransferase 1," Biochemistry, vol. 40, no. 19, pp. 5747-5756, 2001.

[8] H. Naeem, D. Cheng, Q. Zhao et al., "The activity and stability of the transcriptional coactivator $\mathrm{p} / \mathrm{CIP} / \mathrm{SRC}-3$ are regulated by CARM1-dependent methylation," Molecular and Cellular Biology, vol. 27, no. 1, pp. 120-134, 2007.

[9] W. Xu, H. Chen, K. Du et al., "A transcriptional switch mediated by cofactor methylation," Science, vol. 294, no. 5551, pp. 25072511, 2001.

[10] S. Frietze, M. Lupien, P. A. Silver, and M. Brown, "CARM1 regulates estrogen-stimulated breast cancer growth through upregulation of E2F1," Cancer Research, vol. 68, no. 1, pp. 301-306, 2008.

[11] J. Lee and M. T. Bedford, "PABP1 identified as an arginine methyltransferase substrate using high-density protein arrays," EMBO Reports, vol. 3, no. 3, pp. 268-273, 2002.

[12] D. Cheng, J. Côté, S. Shaaban, and M. T. Bedford, "The arginine methyltransferase CARM1 regulates the coupling of transcription and mRNA processing," Molecular Cell, vol. 25, no. 1, pp. 71-83, 2007.

[13] D. Chen, M. Ma, H. Hong et al., "Regulation of transcription by a protein methyltransferase," Science, vol. 284, no. 5423, pp. 2174-2177, 1999.

[14] N. Yadav, J. Lee, J. Kim et al., "Specific protein methylation defects and gene expression perturbations in coactivatorassociated arginine methyltransferase 1-deficient mice," Proceedings of the National Academy of Sciences of the United States of America, vol. 100, no. 11, pp. 6464-6468, 2003.

[15] L. P. Vu, F. Perna, L. Wang et al., "PRMT4 blocks myeloid differentiation by assembling a Methyl-RUNX1-dependent repressor complex," Cell Reports, vol. 5, no. 6, pp. 1625-1638, 2013.

[16] H. Hong, C. Kao, M.-H. Jeng et al., "Aberrant expression of CARM1, a transcriptional coactivator of androgen receptor, in the development of prostate carcinoma and androgenindependent status," Cancer, vol. 101, no. 1, pp. 83-89, 2004.

[17] R. Elakoum, G. Gauchotte, A. Oussalah et al., "CARM1 and PRMT1 are dysregulated in lung cancer without hierarchical features," Biochimie, vol. 97, no. 1, pp. 210-218, 2014.

[18] C. Y. Ou, M. J. LaBonte, P. C. Manegold et al., "A coactivator role of CARM1 in the dysregulation of beta-catenin activity in colorectal cancer cell growth and gene expression," Molecular Cancer Research, vol. 9, no. 5, pp. 660-670, 2011.

[19] A. Mai, D. Cheng, M. T. Bedford et al., "Epigenetic multiple ligands: mixed histone/protein methyltransferase, acetyltransferase, and class III deacetylase (Sirtuin) inhibitors," Journal of Medicinal Chemistry, vol. 51, no. 7, pp. 2279-2290, 2008. 
[20] D. Cheng, S. Valente, S. Castellano et al., "Novel 3,5-bis(bromohydroxybenzylidene)piperidin-4-ones as coactivator-associated arginine methyltransferase 1 inhibitors: enzyme selectivity and cellular activity," Journal of Medicinal Chemistry, vol. 54, no. 13, pp. 4928-4932, 2011.

[21] A. V. Purandare, Z. Chen, T. Huynh et al., "Pyrazole inhibitors of coactivator associated arginine methyltransferase 1 (CARM1)," Bioorganic \& Medicinal Chemistry Letters, vol. 18, pp. 44384441, 2008.

[22] T. Huynh, Z. Chen, S. Pang et al., "Optimization of pyrazole inhibitors of Coactivator Associated Arginine Methyltransferase 1 (CARM1)," Bioorganic \& Medicinal Chemistry Letters, vol. 19, no. 11, pp. 2924-2927, 2009.

[23] M. Allan, S. Manku, E. Therrien et al., "N-Benzyl-1-heteroaryl3-(trifluoromethyl)- $1 H$-pyrazole-5-carboxamides as inhibitors of co-activator associated arginine methyltransferase 1 (CARM1)," Bioorganic \& Medicinal Chemistry Letters, vol. 19, no. 4, pp. 1218-1223, 2009.

[24] E. Therrien, G. Larouche, S. Manku et al., "1,2-Diamines as inhibitors of co-activator associated arginine methyltransferase 1 (CARM1)," Bioorganic and Medicinal Chemistry Letters, vol. 19, no. 23, pp. 6725-6732, 2009.

[25] H. Wan, T. Huynh, S. Pang et al., "Benzo[d]imidazole inhibitors of Coactivator Associated Arginine Methyltransferase 1 (CARM1)-hit to lead studies," Bioorganic \& Medicinal Chemistry Letters, vol. 19, no. 17, pp. 5063-5066, 2009.

[26] J. S. Sack, S. Thieffine, T. Bandiera et al., "Structural basis for CARM1 inhibition by indole and pyrazole inhibitors," The Biochemical Journal, vol. 436, no. 2, pp. 331-339, 2011.

[27] B. R. Selvi, K. Batta, A. H. Kishore et al., "Identification of a novel inhibitor of Coactivator-associated Arginine Methyltransferase 1 (CARM1)-mediated methylation of histone H3 Arg-17," The Journal of Biological Chemistry, vol. 285, no. 10, pp. 7143-7151, 2010.

[28] H. Hu, K. Qian, M.-C. Ho, and Y. G. Zheng, "Small molecule inhibitors of protein arginine methyltransferases," Expert Opinion on Investigational Drugs, vol. 25, no. 3, pp. 335-358, 2016.

[29] L. Li, R. Zhou, H. Geng et al., "Discovery of two aminoglycoside antibiotics as inhibitors targeting the menin-mixed lineage leukaemia interface," Bioorganic \& Medicinal Chemistry Letters, vol. 24, no. 9, pp. 2090-2093, 2014.

[30] S. Chen, Y. Wang, W. Zhou et al., "Identifying novel selective non-nucleoside DNA methyltransferase 1 inhibitors through docking-based virtual screening," Journal of Medicinal Chemistry, vol. 57, no. 21, pp. 9028-9041, 2014.

[31] W. W. Yue, M. Hassler, S. M. Roe, V. Thompson-Vale, and L. H. Pearl, "Insights into histone code syntax from structural and biochemical studies of CARM1 methyltransferase," The EMBO Journal, vol. 26, no. 20, pp. 4402-4412, 2007.

[32] N. Troffer-Charlier, V. Cura, P. Hassenboehler, D. Moras, and J. Cavarelli, "Functional insights from structures of coactivatorassociated arginine methyltransferase 1 domains," The EMBO Journal, vol. 26, no. 20, pp. 4391-4401, 2007.

[33] P. A. Boriack-Sjodin, L. Jin, S. L. Jacques et al., "Structural insights into ternary complex formation of human CARM1 with various substrates," ACS Chemical Biology, vol. 11, no. 3, pp. 763771, 2016.

[34] R. T. Sauer, D. N. Bolon, B. M. Burton et al., "Sculpting the proteome with $\mathrm{AAA}(+)$ proteases and disassembly machines," Cell, vol. 119, no. 1, pp. 9-18, 2004.
[35] C. A. Lipinski, F. Lombardo, B. W. Dominy, and P. J. Feeney, "Experimental and computational approaches to estimate solubility and permeability in drug discovery and development settings," Advanced Drug Delivery Reviews, vol. 46, no. 1-3, pp. 3-26, 2001.

[36] J. B. Baell and G. A. Holloway, "New substructure filters for removal of pan assay interference compounds (PAINS) from screening libraries and for their exclusion in bioassays," Journal of Medicinal Chemistry, vol. 53, no. 7, pp. 2719-2740, 2010.

[37] A. Whitty, "Growing PAINS in academic drug discovery," Future Medicinal Chemistry, vol. 3, no. 7, pp. 797-801, 2011.

[38] J. Baell and M. A. Walters, "Chemistry: chemical con artists foil drug discovery," Nature, vol. 513, no. 7519, pp. 481-483, 2014.

[39] Accelrys Software Inc, Pipeline Pilot. Version 7.5, Accelrys Software Inc, San Diego, Calif, USA, 2008.

[40] D. Frees, A. Chastanet, S. Qazi et al., "Clp ATPases are required for stress tolerance, intracellular replication and biofilm formation in Staphylococcus aureus," Molecular Microbiology, vol. 54, no. 5, pp. 1445-1462, 2004.

[41] O. Gaillot, S. Bregenholt, F. Jaubert, J. P. Di Santo, and P. Berche, "Stress-induced ClpP serine protease of Listeria monocytogenes is essential for induction of listeriolysin $\mathrm{O}$-dependent protective immunity," Infection and Immunity, vol. 69, no. 8, pp. 49384943, 2001

[42] R. A. Friesner, R. B. Murphy, M. P. Repasky et al., "Extra precision glide: docking and scoring incorporating a model of hydrophobic enclosure for protein-ligand complexes," Journal of Medicinal Chemistry, vol. 49, no. 21, pp. 6177-6196, 2006.

[43] J. Wang, J. A. Hartling, and J. M. Flanagan, "The structure of ClpP at $2.3 \AA$ resolution suggests a model for ATP- dependent proteolysis," Cell, vol. 91, no. 4, pp. 447-456, 1997.

[44] T. J. A. Ewing, S. Makino, A. G. Skillman, and I. D. Kuntz, "DOCK 4.0: search strategies for automated molecular docking of flexible molecule databases," Journal of Computer-Aided Molecular Design, vol. 15, no. 5, pp. 411-428, 2001.

[45] D. S. Goodsell, G. M. Morris, and A. J. Olson, "Automated docking of flexible ligands: applications of AutoDock," Journal of Molecular Recognition, vol. 9, no. 1, pp. 1-5, 1996.

[46] J. Zhang and Y. G. Zheng, "SAM/SAH analogs as versatile tools for SAM-dependent methyltransferases," ACS Chemical Biology, vol. 11, no. 3, pp. 583-597, 2016.

[47] R. M. Raju, A. L. Goldberg, and E. J. Rubin, "Bacterial proteolytic complexes as therapeutic targets," Nature Reviews Drug Discovery, vol. 11, no. 10, pp. 777-789, 2012.

[48] X. Zhang and X. Cheng, "Structure of the predominant protein arginine methyltransferase PRMT1 and analysis of its binding to substrate peptides," Structure, vol. 11, no. 5, pp. 509-520, 2003. 

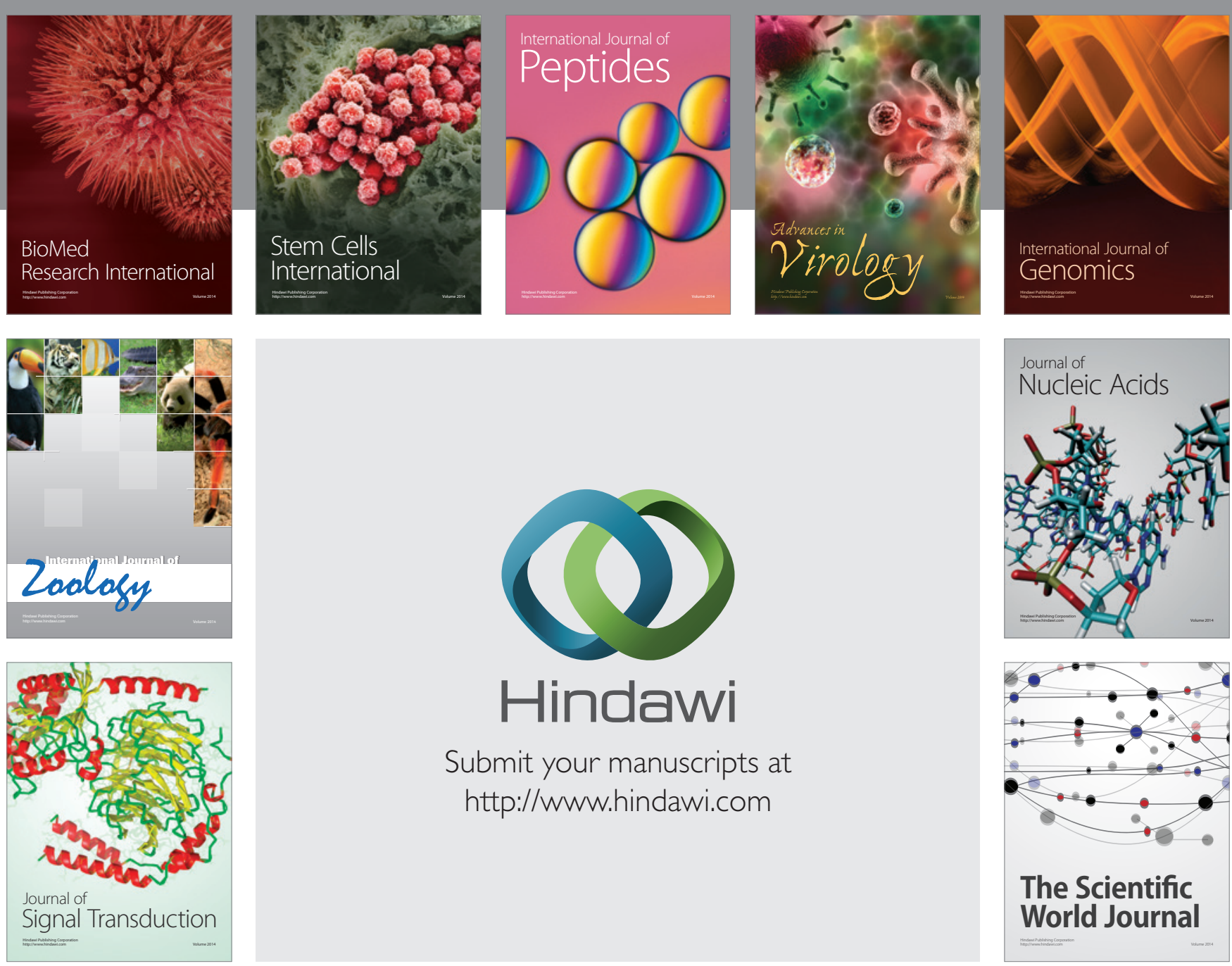

Submit your manuscripts at

http://www.hindawi.com
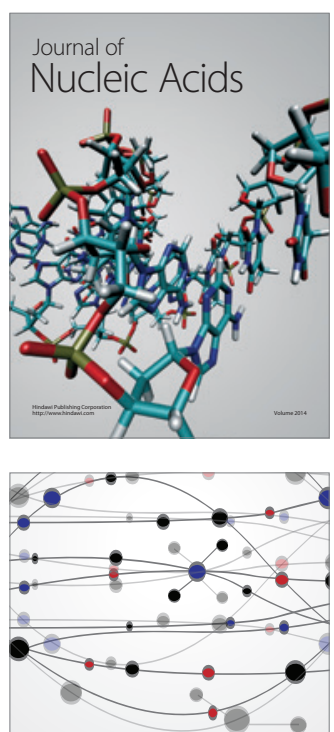

The Scientific World Journal
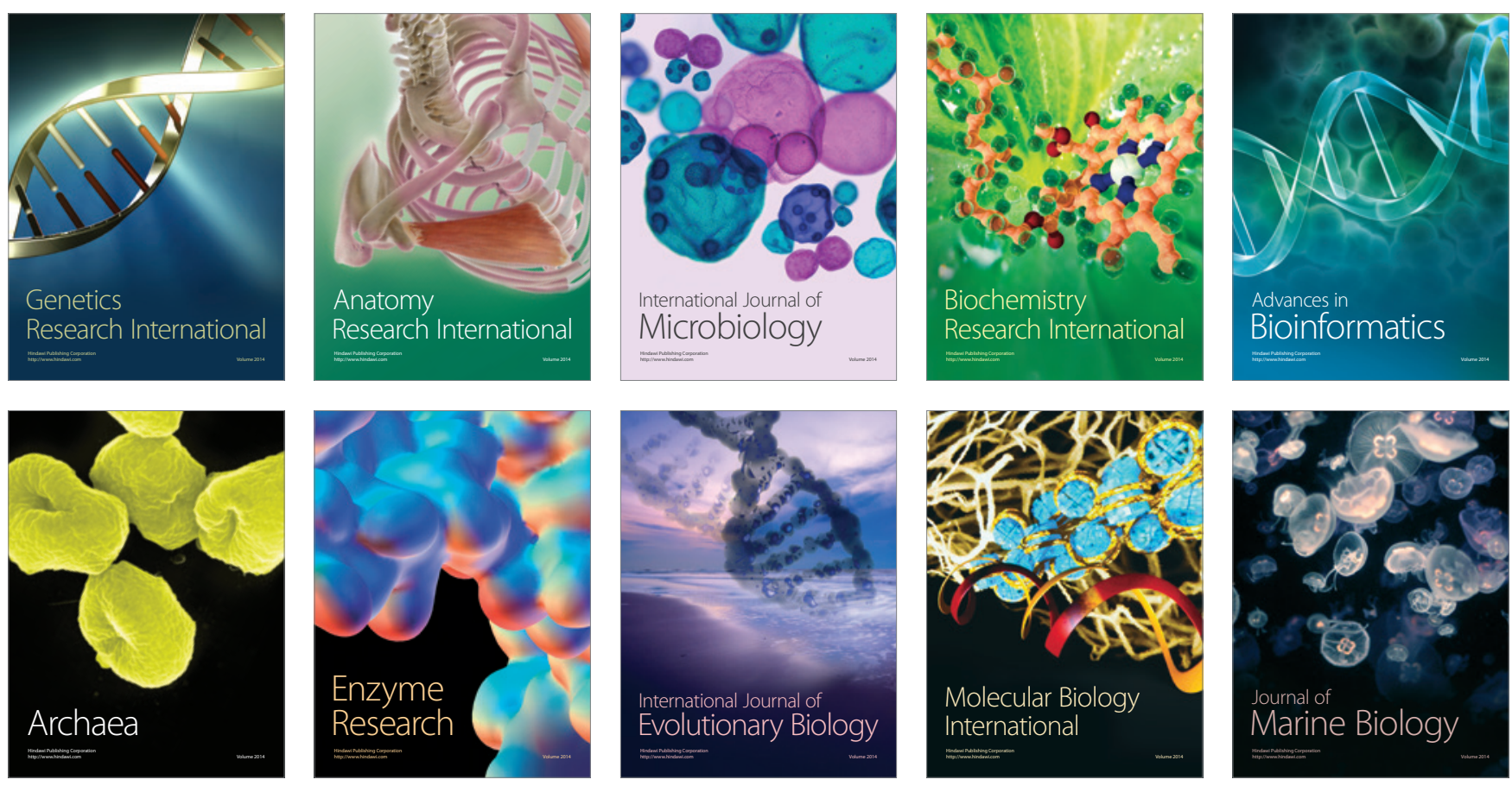